\title{
Parámetros productivos y hematológicos de Piaractus brachypomus suplementados con ácido ascórbico y sometidos a estrés por hipoxia
}

\author{
Productive and hematological parameters of Piaractus \\ brachypomus supplemented with ascorbic acid and subjected to \\ hypoxia stress
}

\section{Parámetros produtivos e hematológicos de Piaractus brachypomus suplementados com ácido ascórbico e submetidos ao estresse pela hipóxia}

\author{
Adriana S. Corredor-Castillo ${ }^{1 *}$, Miguel A. Landines-Parra ${ }^{2 *}$ \\ 1 Zoot, MSc, PhD; \\ 2 Zoot, PhD \\ * Laboratorio de Fisiología de Peces, Facultad de Medicina Veteriaria y de Zootecnia, Universidad Nacional de Colombia, \\ Bogotá, Colombia \\ Email: ascorredorc@unal.edu.co
}

Recibido: 30 de enero de 2017

Aceptado: 26 de febrero de 2018

\begin{abstract}
Resumen
Se evaluó el efecto de la suplementación con vitamina C sobre algunos parámetros productivos y hematológicos de cachama blanca sometida a estrés por hipoxia aguda. Fueron utilizados 72 juveniles $(30 \pm 4,76 \mathrm{~g})$,los cuales fueron alimentados ad libitum durante 30 días con dieta de $32 \%$ de proteína y diferentes niveles de vitamina C: T1:0 mg/ $/ \mathrm{kg}$; T2:100 mg/kg; T3:200mg/kg; T4:400 mg/kg; T5:800 mg/kg; T6:1600 mg/kg. El día 30, los animales fueron sometidos a exposición aérea durante 60 segundos y devueltos al agua. Se tomaron muestras de sangre a los 5, 30, 60 y 360 minutos, para determinación de hematocrito, hemoglobina, recuento celular (rojas y blancas), conteo diferencial de leucocitos, volumen corpuscular medio (VCM), hemoglobina corpuscular media (HCM) y concentración de hemoglobina corpuscular media (CHCM). Los pesces fueron pesados para calcular ganacia de peso (GP), tasa de crecimiento específico (TCE), crecimiento relativo (CR) y supervivencia. Los datos fueron sometidos a análisis de varianza (ANAVA) y las medias comparadas por prueba de Tukey (5\%). Hubo diferencias significativas para GP, TCE y CR, parámetros en los que el T2 fue el mejor. Asimismo, hubo diferencias significativas para los monocitos entre T1 y T4, T5 y T6 en el primer muestreo, mientras que para basófilos, linfocitos, neutrófilos y eosinófilos no se observaron diferencias entre tratamientos ni tiempos. La supervivencia fue del $100 \%$ en todos los tratamientos. Los resultados sugieren que la suplementación con vitamina C en la dieta podría favorecer el crecimiento de la especie, pero que niveles excesivos $(1600 \mathrm{mg} / \mathrm{kg})$ pueden tener efectos adversos. Por otro lado, aunque hubo diferencias significativas entre tratamientos en el número de leucocitos y el porcentaje de monocitos, los parametros hematológicos evaluados permanecieron constantes ante la condición de hipoxia aguda, independientemente del nivel de consumo de la vitamina.
\end{abstract}


Palabras clave: ácido ascórbico, hipoxia, parámetros hematológicos, parámetros productivos, Piaractus brachypomus.

\begin{abstract}
This experiment evaluated the effect of the supplementation of vitamin $C$ on some productive and hematological parameters of cachama blanca submitted to acute hypoxia. 72 juveniles $(30 \pm 4.76 \mathrm{~g})$ were used. They were fed ad libitum during 30 days with a diet of $32 \%$ of protein and different levels of vitamin C as follows: T1:0 mg $/ \mathrm{kg} ;$ T2:100 mg/ $/ \mathrm{kg}$; T3:200mg/kg; $\mathrm{T} 4: 400 \mathrm{mg} / \mathrm{kg} ; \mathrm{T} 5: 800 \mathrm{mg} / \mathrm{kg} ; \mathrm{T} 6: 1600 \mathrm{mg} / \mathrm{kg}$. On day 30 the animals were sujected to aerial exposition during 60 seconds and returned to the water. Blood samples were taken at 5, 30, 60 and 360 minutes, to determine hematocrit, hemoglobin, cell count (red and white), leukocytes differential count, mean corpuscular volume, (MCV), mean corpuscular hemoglobin $(\mathrm{MCH})$ and mean corpuscular hemoglobin concentration $(\mathrm{MCHC})$. All animals were weighted to calculate weight growth (WG), specific growth rate (SGR), and relative growth (RG). Finally, survival rate was determined. The data were analyzed using variance analysis (ANOVA) and the means were compared using the Tukey's test (5\%). There were significant differences for WG, SGR, RG. T2 had the best parameters. The number of leukocytes was significatively higher in T5 in all sampling times. There were significative differences for monocytes among T1 and T4, and T5 and T6 in the first sampling, while for the basophils, lymphocytes, neutrophils and eosinophils there were not any differences among treatments or sampling times. Survival was $100 \%$ in all treatments. The results sugest that the supplementation with vitamine $\mathrm{C}$ in the diet could favour the growth of the species (WG, SGR, RG), but that excesive levels $(1600 \mathrm{mg} / \mathrm{kg}$ ) could have adverse effects on these parameter. On the other hand, although there were significative differences among treatments in the number of leukocytes and the percentage of monocytes, the hematological parameters evaluated, remained constant in relation to the condition of acute hypoxia, independently of the level of vitamin consumption.
\end{abstract}

Key words: ascorbic acid, hypoxia, hematological parameters, productive parameters, Piaractus brachypomus.

\title{
Resumo
}

Foi avaliado o efeito da suplementação com vitamina $\mathrm{C}$ em alguns parâmetros produtivos e hematológicos da cachama branca submetida à hipóxia aguda. Foram utilizados 72 juvenis $(30 \pm 4,76 \mathrm{~g})$, que foram alimentados ad libitum por 30 dias com uma dieta de $32 \%$ de proteína e diferentes níveis de vitamina C, constituindo os seguintes tratamentos: T1:0 mg/kg; $\mathrm{T} 2: 100 \mathrm{mg} / \mathrm{kg}$; T3:200 mg/kg; T4:400 mg/kg; T5:800 mg/kg; T6:1600 mg/kg. No dia 30, os animais foram submetidos a exposição ao ar durante 60 segundos e devolvidos para a água. Amostras de sangue foram coletadas aos 5; 30; 60 e 360 minutos, para determinação do hematócrito, hemoglobina, contagem de células (vermelhas e brancas), contagem diferencial de leucócitos, volume corpuscular médio (VCM), hemoglobina corpuscular média (HCM) e concentração de hemoglobina corpuscular média (CHCM). Todos os animais foram pesados para calcular o ganho de peso (GP), taxa de crescimento específico (TCE) e crescimento relativo (CR). Finalmente foi determinada a sobrevivência. Os dados foram submetidos à análise de variância (ANOVA) e as médias comparadas pelo teste de Tukey (5\%). Houve diferenças significativas para os parâmetros GP, TCE e CR em que T2 foi o melhor. O número de leucócitos foi significativamente maior no T5 em todos os tempos de amostragem. Houve também diferenças significativas para monócitos entre T1 e T4, T5 e T6 na primeira amostragem, enquanto para basófilos, linfócitos, neutrófilos e eosinófilos não foram observadas diferenças entre os tratamentos e os tempos. A sobrevivência foi de $100 \%$ em todos os tratamentos. Os resultados sugerem que a suplementação com vitamina C na dieta pode promover o crescimento da espécie (GP, TCE e CR), porém, níveis excessivos (1600 mg/ kg) podem ter efeitos adversos sobre esse parâmetro. Por outro lado, embora houvesse diferenças significativas entre os tratamentos no número de leucócitos e na percentagem de monócitos, aparentemente tal suplementação não parece ter efeito sobre os parâmetros hematológicos avaliados, os quais em geral permaneceram constantes em condições de hipoxia aguda, independente do nível de vitamina consumida.

Palavras chave: ácido ascórbico, hipoxia, parâmetros hematológicos, parâmetros produtivos, Piaractus brachypomus.

\section{Introducción}

Actualmente la acuicultura es una de las principales fuentes de proteína para la seguridad alimentaria a nivel mundial. Adicionalmente constituye una actividad generadora de empleo en la mayoría de países en desarrollo (FAO, 2018). Según FAO (2016) es el sistema de producción animal que ha presentado mayor crecimiento en los últimos años.

Para que dicho crecimiento sea posible, ha sido necesario implementar sistemas de producción cada vez más intensivos, en los que los organismos cultivados pueden ser expuestos a factores estresantes propios de dichos sistemas, como por ejemplo problemas de calidad de agua, alta densidad de siembra, captura y traslado, entre otros, los cuales pueden ocasionar bajas de oxígeno y en ocasiones enfermedades o hasta la muerte de los ejemplares (Urbinati y Carneiro, 2005). Por tal razón, es necesario generar alternativas que permitan disminuir el impacto de los agentes estresantes, inevitables dentro del proceso productivo, 
que garanticen el bienestar de los animales durante su ciclo de producción.

Diferentes estudios demuestran que el ácido ascórbico (vitamina $\mathrm{C}$ ) tiene funciones importantes en los procesos fisiológicos de varias especies de peces (Chagas y Val, 2003). Es un nutriente correlacionado con el funcionamiento del sistema inmune, constituye un cofactor implicado en reacciones fisiológicas como la hidroxilación de los aminoácidos prolina y lisina, responsables de la síntesis de colágeno (Falcon et al., 2007; Drouin et al., 2011), en la síntesis de neurotransmisores como la serotonina y en la transformación de la dopamina en noradrenalina (Miyasaki et al., 1995; Chagas y Val, 2003; Urbinati y Carneiro, 2005), adicionalmente, juega un papel importante en el metabolismo del hierro (Ortuño et al., 2001). Sin embargo, en condiciones naturales los peces teleósteos, en especial los de agua dulce, no son capaces de sintetizar tal vitamina, teniendo que satisfacer su demanda obteniéndola del alimento consumido (Drouin et al., 2011) motivo por el cual, en cautiverio es imprescindible suministrarla en la dieta.

Peces alimentados con dietas deficientes en ácido ascórbico (A.A) presentan deformidades en las estructuras de soporte de las branquias y los ojos y en las aletas y la columna (Darias et al., 2011), así como una disminución en los valores de hematocrito (Miyasaki et al., 1995; Verlhac et al.; 1998; Chagas y Val, 2003) y de hemoglobina, pues la deficiencia ocasiona una reducción en la capacidad de absorber y redistribuir el hierro, con la consecuente disminución en la capacidad de sintetizarla (Verlhac et al., 1998; Abreu y Urbinati, 2006).

En situaciones de estrés, los peces presentan como respuesta primaria mayor actividad de las hormonas equivalentes a las de la corteza adrenal de otros vertebrados (Valenzuela et al., 2002) y posteriormente, como respuesta secundaria, presentan alteraciones en parámetros como el conteo de células sanguíneas, haciendo que este parámetro sea útil en la determinación de la respuesta de los peces ante situaciones adversas (Wendelaar-Bonga, 1997; Sloman et al., 2001). Finalmente, como respuesta terciaria, los animales disminuyen su tasa de crecimiento y su desempeño productivo, al tiempo que se hacen más susceptibles a adquirir enfermedades (Barton e Iwama, 1991; Wendelaar-Bonga, 1997; Barnett y Pankhurst, 1998).

Por lo anterior, el objetivo del presente trabajo fue evaluar el efecto de la suplementación en la dieta con ácido ascórbico, sobre algunos parámetros productivos y hematológicos de juveniles de cachama blanca, Piaractus brachypomus sometidos a estrés por hipoxia aguda.

\section{Materiales y métodos}

\section{Localización}

El trabajo fue desarrollado en el Laboratorio de Ictiología de la Facultad de Medicina Veterinaria y de Zootecnia de la Universidad Nacional de Colombia, ubicado en la ciudad de Bogota, a 2.600 msnm, bajo condiciones controladas de laboratorio $\left(24^{\circ} \mathrm{C}\right)$. Los análisis de laboratorio fueron realizados en los laboratorios Clínico y de Nutrición Animal de la misma facultad.

\section{Animales experimentales}

Fueron utilizados 72 juveniles de cachama blanca con peso promedio de $30 \pm 4,76 \mathrm{~g}$, los cuales fueron mantenidos en 24 acuarios de 80 litros a una carga inicial de 1,2 g/L y alimentados ad libitum (consumo aproximado: 3,86\% de la biomasa/día) durante 30 días con una dieta extruida de $32 \%$ de proteína, cuya composición proximal se presenta en la Tabla 1. La Composición de la dieta experimental formulada con $32 \%$ de proteína se muestra en la Tabla 2.

La mezcla formulada y realizada en el laboratorio, sin adición de A.A, fue dividida en seis porciones, a cada una de las cuales se le adicionó un nivel diferente de A.A bajo la forma de L-ascorbil-2-monofosfato (Rovimix Stay-C35 $\left.{ }^{\circledR}\right)$, constituyendo los siguientes tratamientos: T1: 0 mg/kg; T2: 100 mg/kg; T3: 200 mg/kg; T4: 400 $\mathrm{mg} / \mathrm{kg}$; T5: $800 \mathrm{mg} / \mathrm{kg}$; T6: $1600 \mathrm{mg} / \mathrm{kg}$.

Las materias primas seleccionadas y las dietas extruidas fueron sometidas a análisis proximal, en el Laboratorio de Nutrición Animal de la Facultad de Medicina Veterinaria y de Zootecnia de la Universidad Nacional de Colombia, de acuerdo con los métodos oficiales de Análisis de la Asociación de Química Analítica (AOAC, 2012).

\section{Obtención y manejo de material biológico}

Finalizada la fase de alimentación (30 días), los animales fueron sometidos a hipoxia aguda, consistente en una exposición aérea durante 60 segundos e inmediata devolución al agua. Previa anestesia con metasulfonato de tricaina (75 ppm), se realizaron colectas de sangre mediante punción en la vena caudal de 3 individuos por tratamiento a los 5, 30, 60 y 360 minutos después de haber sido devueltos al agua. Los peces fueron seleccionados aleatoriamente de los diferentes acuarios de cada tratamiento para cada tiempo de muestreo y sacrificados mediante sobredosis de anestesia. Previo al experimento se obtuvo el aval del comité de bioética de la Facultad de Medicina Vete- 
Tabla 1. Composición proximal de la dieta experimental (expresada como alimento)

\begin{tabular}{|c|c|c|c|c|c|}
\hline \% MS* & \% Proteína & \% E.E** & \% Fibra cruda & \% Cenizas & \% ENN \\
\hline $91,55 \pm 0,03$ & $32,28 \pm 0,49$ & $1,97 \pm 0,37$ & $2,27 \pm 0,18$ & $2,87 \pm 0.01$ & $55,29 \pm 0,04$ \\
\hline
\end{tabular}

*Materia seca; ${ }^{*}$ Extracto etéreo; ${ }^{* *}$ Extracto no nitrogenado.

Tabla 2. Composición de la dieta experimental formulada con $32 \%$ de proteína en base seca

\begin{tabular}{|l|c|}
\hline \multicolumn{1}{|c|}{ Ingrediente } & \% de inclusión \\
\hline Harina de carne & 11,22 \\
\hline Harina de pescado 64 & 4,00 \\
\hline Torta de soya 48 & 27,00 \\
\hline Arroz molido & 12,00 \\
\hline Maíz amarillo & 28,75 \\
\hline Gluten de maíz & 6,92 \\
\hline Mogolla de trigo & 5,65 \\
\hline Aceite de soya & 1,00 \\
\hline NaCl & 0,30 \\
\hline Fosfato bicálcico & 1,42 \\
\hline Premezcla vitaminas (libre de A.A.) & 0,25 \\
\hline Premezcla minerales & 0,30 \\
\hline Metionina & 0,01 \\
\hline Lisina & 0,08 \\
\hline Cl Colina & 0,10 \\
\hline CMC & 1,00 \\
\hline
\end{tabular}

rinaria y de Zootecnia de la Universidad Nacional de Colombia.

Todos los individuos fueron pesados para calcular ganancia de peso (GP), tasa de crecimiento específico (TCE) y crecimiento relativo (CR), mediante ecuaciones descritas por Ricker (1975):

Ganancia de peso g (GP): Peso final-Peso inicial

Tasa de crecimiento específico \%/día (TCE): [(Ln peso final-Ln peso inicial) /Tiempo] ${ }^{\star} 100$

Crecimiento relativo (CR): Ganancia de peso/Peso inicial $^{*} 100$

Con las muestras de sangre obtenidas se tomó una primera porción en capilares de $50 \mu \mathrm{L}$, los cuales fueron centrifugados durante 5 minutos a $5000 \mathrm{rpm}$ para la determinación del hematocrito. Una segunda porción se utilizó para la determinación de hemoglobina, la cual se realizó utilizando el kit comercial Drabkin
Spinreact ${ }^{\circledR}$, mediante el uso de espectrofotómetro Stat $\operatorname{Fax}^{\circledR} 3300$.

Para conteo óptico de glóbulos rojos y blancos mediante cámara de Neubauer se llevó a cabo previamente una dilución de 1:100, con reactivo de Natt Herricks, también se realizó un extendido en portaobjeto de vidrio para recuento diferencial de células blancas mediante tinción de Wright. Con estos parámetros se calcularon: volumen corpuscular medio (VCM), hemoglobina corpuscular media (HCM) y concentración de hemoglobina corpuscular media ( $\mathrm{CHCM})$, mediante la metodología descrita por Conroy (1998):

$$
\begin{aligned}
& V C M=\% \text { Hematocrito }{ }^{*} 10 / N^{\circ} \text { eritrocitos } \\
& H C M=\text { Hemoglobina }{ }^{*} 10 / N^{\circ} \text { eritrocitos } \\
& C H C M=\text { Hemoglobina }{ }^{*} 100 / \% \text { hematocrito }
\end{aligned}
$$

La sobrevivencia fue calculada mediante la siguiente ecuación:

$$
\begin{gathered}
\% \text { Sobrevivencia: } \mathrm{S}=\left(\mathrm{N}^{\circ} \text { final de individuos } /\right. \\
\left.\mathrm{N}^{\circ} \text { inicial de individuos }\right){ }^{*} 100
\end{gathered}
$$

\section{Análisis estadístico}

El experimento tuvo un diseño experimental completamente al azar con arreglo de parcelas divididas en el tiempo. Se comprobó el cumplimiento de los supuestos estadísticos de normalidad de los datos e independencia del error. Para verificar la existencia de diferencias significativas $(p<0,05)$ entre los tratamientos se realizó un análisis de varianza (ANAVA) y en los casos que se encontraron, las medias fueron comparadas mediante la prueba de Tukey (5\%). Los análisis se realizaron mediante la utilización del software SAS System V9.0 ${ }^{\circledR}$.

\section{Resultados}

Los valores de GP, TCE y CR se presentan en la Tabla 3 , mostrando que hubo diferencias significativas para las tres variables. Los ejemplares del tratamiento con $100 \mathrm{mg} / \mathrm{kg}$ (T2) presentaron valores significativamente mayores cuando se compararon con los demás tratamientos; los ejemplares suplementados con 1600 mg/ kg (T6) presentaron el menor desempeño productivo 
respecto a los demás tratamientos. La supervivencia fue del $100 \%$ en todos los tratamientos.

El hematocrito y la hemoglobina no presentaron diferencias significativas $(p>0,05)$, ni entre tratamientos ni entre tiempos (Tabla 4). Lo mismo fue encontrado para conteo de células rojas, VCM, HCM y CHCM. En el recuento diferencial no hubo diferencias significativas en el porcentaje de linfocitos, basófilos, neutrófilos y eosinófilos, ni entre tratamientos ni entre tiempos de muestreo. Solamente en el caso de los monocitos se presentaron diferencias significativas en el primer tiempo de muestreo, observándose un porcentaje mayor de estas células en los ejemplares del T1, con respecto a los tratamientos T4, T5 y T6 (Tabla 5). En el caso de los glóbulos blancos se observó que los ejemplares suplementados con $800 \mathrm{mg} / \mathrm{kg}$ presentaron un mayor número de células que los demás tratamientos en todos los tiempos de muestreo (Figura 1).

\section{Discusión}

La mayor ganancia de peso encontrada al al suplementar los animales con $100 \mathrm{mg} / \mathrm{kg}$ de A.A en la dieta con respecto al control indica que la inclusión dietaria sería recomendable para atender los requerimientos para crecimiento de los juveniles de la especie. Estos resultados coinciden con lo reportado por $\mathrm{Ai}$ et al., (2004) en Lateolabrax japonicus, Ming et al., (2012) en Megalobrama amblycephala, Zhou et al., (2012) en Rachycentron canadum, quienes demostraron que al suplementar la dieta con vitamina C, los individuos presentaban mejor ganancia de peso y tasa de crecimiento específico; lo anterior también coincide con los resultados reportados por Martins (1998) en Piaractus mesopotamicus y Chagas y Val (2003) en Colossoma macropomum, quienes observaron que la suplemen- tación en la dieta con ácido ascórbico mejoraba el desempeño zootécnico de los peces. No obstante, algunos autores afirman que no existen diferencias significativas en el desempeño productivo de los peces al ser suplementados con vitamina C (Navarro et al., 2010; Reis et al., 2011; Notash, 2012, Zaminham et al., 2012; Gao et al., 2014).

De hecho, aun existe controversia respecto al uso o al nivel de inclusión de la vitamina $\mathrm{C}$ en la dieta. En el presente trabajo el mejor nivel fue $100 \mathrm{mg} / \mathrm{kg}$, resultado que sería consistente con lo reportado por Chen et al., (2015) en Micropterus salmoides, Liang et al., (2017) en Pelteobagrus fulvidraco y Arab e Islami (2015) en Salmo trutta, quienes afirman que niveles de

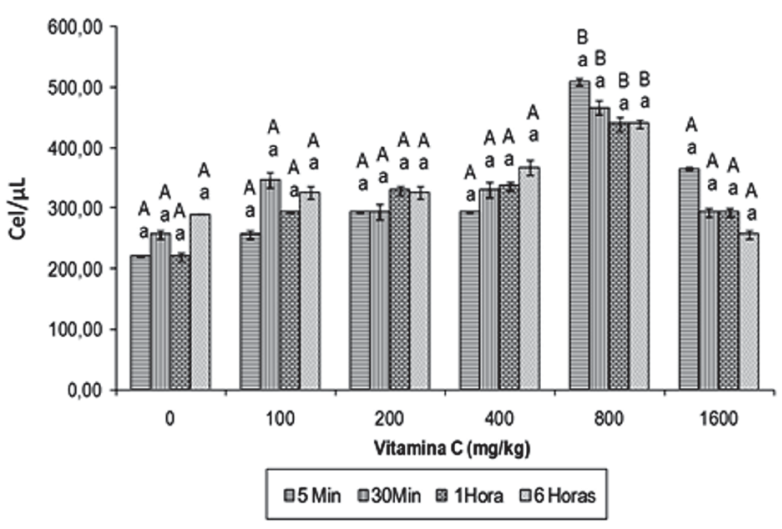

Figura 1. Cantidad de glóbulos blancos $(\mathrm{Cel} / \mu \mathrm{L})$ de cachama blanca alimentada con seis niveles de inclusión de ácido ascorbico dietario y cuatro tiempos de muestreo pos hipoxia aguda. Letras mayúsculas diferentes indican diferencia significativa $(p<0,05)$ entre niveles; letras minúsculas diferentes indican diferencia significativa $(p<0,05)$ entre tiempos.

Tabla 3. Valores medios \pm D. S. de ganancia de peso (GP), crecimiento relativo (CR) y tasa de crecimiento específico (TCE) de cachama blanca alimentada con diferentes niveles de inclusión de ácido ascorbico dietario

\begin{tabular}{|c|c|c|c|}
\hline Tratamiento & GP $(\mathbf{g})$ & CR $(\%)$ & TCE (\% día) \\
\hline T1 & $19,85 \pm 3,62^{\mathrm{b}}$ & $63,73 \pm 0,15^{\mathrm{b}}$ & $1,63 \pm 0,26^{\mathrm{bc}}$ \\
\hline T2 & $22,88 \pm 3,37^{\mathrm{a}}$ & $76,73 \pm 0,15^{\mathrm{a}}$ & $1,90 \pm 0,18^{\mathrm{a}}$ \\
\hline T3 & $20,01 \pm 4,09^{\mathrm{b}}$ & $70,76 \pm 0,16^{\mathrm{b}}$ & $1,78 \pm 0,30^{\mathrm{b}}$ \\
\hline T4 & $20,04 \pm 4,29^{\mathrm{b}}$ & $69,99 \pm 0,17^{\mathrm{b}}$ & $1,77 \pm 0,43^{\mathrm{b}}$ \\
\hline T5 & $19,20 \pm 4,04^{\mathrm{b}}$ & $70,86 \pm 0,16^{\mathrm{b}}$ & $1,79 \pm 0,29^{\mathrm{b}}$ \\
\hline T6 & $17,23 \pm 2,99^{\mathrm{c}}$ & $56,33 \pm 0,14^{\mathrm{c}}$ & $1,49 \pm 0,28^{\mathrm{c}}$ \\
\hline
\end{tabular}

Letras diferentes dentro de las columnas indican diferencias significativas $(p<0,05)$.

T1:0 mg/kg; T2:100 mg/kg; T3:200mg/kg; T4:400 mg/kg; T5:800 mg/kg; T6:1600 mg/kg. 
Tabla 4. Valores de hematocrito y hemoglobina (Media \pm D. S.) de cachama blanca alimentada con seis niveles de inclusión de ácido ascorbico dietario y cuatro tiempos de muestreo pos hipoxia aguda

\begin{tabular}{|c|c|c|c|c|c|c|c|}
\hline Tiempo & Tratamiento & Hem & crit & $(\%)$ & Hems & in & (dL) \\
\hline \multirow{6}{*}{$5 \min$} & $0 \mathrm{mg} / \mathrm{kg}$ & 37,33 & \pm & 0,58 & 16,58 & \pm & 1,44 \\
\hline & $100 \mathrm{mg} / \mathrm{kg}$ & 37,67 & \pm & 0,58 & 15,27 & \pm & 0,34 \\
\hline & $200 \mathrm{mg} / \mathrm{kg}$ & 36,00 & \pm & 2,00 & 16,30 & \pm & 1,15 \\
\hline & $400 \mathrm{mg} / \mathrm{kg}$ & 37,33 & \pm & 1,15 & 16,45 & \pm & 1,22 \\
\hline & $800 \mathrm{mg} / \mathrm{kg}$ & 36,33 & \pm & 2,08 & 16,45 & \pm & 1,37 \\
\hline & $1600 \mathrm{mg} / \mathrm{kg}$ & 37,00 & \pm & 1,00 & 15,07 & \pm & 0,37 \\
\hline \multirow{6}{*}{$30 \mathrm{~min}$} & $0 \mathrm{mg} / \mathrm{kg}$ & 35,33 & \pm & 2,31 & 16,24 & \pm & 0,37 \\
\hline & $100 \mathrm{mg} / \mathrm{kg}$ & 36,00 & \pm & 2,31 & 15,07 & \pm & 0,39 \\
\hline & $200 \mathrm{mg} / \mathrm{kg}$ & 38,00 & \pm & 2,08 & 15,32 & \pm & 0,57 \\
\hline & $400 \mathrm{mg} / \mathrm{kg}$ & 37,00 & \pm & 2,08 & 15,94 & \pm & 0,68 \\
\hline & $800 \mathrm{mg} / \mathrm{kg}$ & 37,67 & \pm & 1,53 & 15,86 & \pm & 0,16 \\
\hline & $1600 \mathrm{mg} / \mathrm{kg}$ & 35,33 & \pm & 1,15 & 16,05 & \pm & 1,56 \\
\hline \multirow{6}{*}{$60 \mathrm{~min}$} & $0 \mathrm{mg} / \mathrm{kg}$ & 36,67 & \pm & 1,15 & 15,81 & \pm & 1,10 \\
\hline & $100 \mathrm{mg} / \mathrm{kg}$ & 36,33 & \pm & 2,31 & 15,41 & \pm & 1,06 \\
\hline & $200 \mathrm{mg} / \mathrm{kg}$ & 37,67 & \pm & 1,53 & 15,81 & \pm & 0,97 \\
\hline & $400 \mathrm{mg} / \mathrm{kg}$ & 36,00 & \pm & 1,73 & 15,29 & \pm & 1,08 \\
\hline & $800 \mathrm{mg} / \mathrm{kg}$ & 36,00 & \pm & 1,81 & 16,10 & \pm & 0,51 \\
\hline & $1600 \mathrm{mg} / \mathrm{kg}$ & 36,00 & \pm & 1,00 & 15,54 & \pm & 0,19 \\
\hline \multirow{6}{*}{360 minutos } & $0 \mathrm{mg} / \mathrm{kg}$ & 37,33 & \pm & 1,15 & 15,41 & \pm & 0,32 \\
\hline & $100 \mathrm{mg} / \mathrm{kg}$ & 36,33 & \pm & 1,00 & 15,63 & \pm & 0,73 \\
\hline & $200 \mathrm{mg} / \mathrm{kg}$ & 37,00 & \pm & 0,58 & 15,32 & \pm & 0,21 \\
\hline & $400 \mathrm{mg} / \mathrm{kg}$ & 35,67 & \pm & 1,15 & 15,54 & \pm & 0,38 \\
\hline & $800 \mathrm{mg} / \mathrm{kg}$ & 35,67 & \pm & 1,00 & 15,54 & \pm & 0,19 \\
\hline & $1600 \mathrm{mg} / \mathrm{kg}$ & 37,33 & \pm & 2,08 & 15,41 & \pm & 0,40 \\
\hline
\end{tabular}

entre 109 y 177 mg/kg de ácido ascórbico en la dieta serían suficientes para mejorar el desempeño productivo de los animales y que niveles mayores de suplementación no serían necesarios para tal propósito. No obstante, Ibrahem et al., (2010) observaron que Oreochromis niloticus suplementadas con $500 \mathrm{mg} / \mathrm{kg}$ de vitamina $\mathrm{C}$, presentaron mejor ganancia de peso, tasa de crecimiento específico y sobrevivencia que peces no suplementados. Igualmente, Gusmão et al., (2007) pudieron comprobar que la suplementación con 800 $\mathrm{mg} / \mathrm{kg}$ de vitamina $\mathrm{C}$ en la dieta de Brycon amazonicus favoreció la ganancia de peso de los animales. Sin embargo, en el presente estudio, niveles mayores a 100 $\mathrm{mg} / \mathrm{kg}$ de A.A no presentaron efecto positivo sobre dicho parámetro. Por el contrario, a medida que se aumentó el nivel de suplementación, los valores obtenidos disminuyeron, inclusive con diferencias significativas $(p<0,05)$ entre el T6 $(1600 \mathrm{mg} / \mathrm{kg})$ y los demás tratamientos. Este resultado coincide con lo reportado por Ramesha et al., (2000), quienes afirman que la su- 
Tabla 5. Recuento diferencial de células blancas (Media \pm D. S.) de cachama blanca alimentada con seis niveles de inclusión de ácido ascorbico dietario y cuatro tiempos de muestreo pos hipoxia aguda

\begin{tabular}{|c|c|c|c|c|c|c|c|c|c|c|}
\hline \multirow{2}{*}{$\begin{array}{l}\mathbf{0 ~} \mathbf{~ m g} / \mathbf{k g} \\
5 \mathrm{~min}\end{array}$} & \multicolumn{2}{|c|}{ Lin $(\%)$} & \multicolumn{2}{|c|}{ Neu $(\%)$} & \multicolumn{2}{|c|}{ Eos (\%) } & \multicolumn{2}{|c|}{ Mon (\%) } & \multicolumn{2}{|c|}{ Bas (\%) } \\
\hline & $60,67 \pm$ & 8,72 & $6,33 \pm$ & 0,58 & $25,67 \pm$ & 1,37 & $7,33 \pm$ & $0,58^{\mathrm{A}}$ & $0,00 \pm$ & 0,00 \\
\hline $30 \mathrm{~min}$ & $65,67 \pm$ & 1,53 & $5,67 \pm$ & 1,53 & $23,00 \pm$ & 1,00 & $5,67 \pm$ & 0,58 & $0,00 \pm$ & 0,00 \\
\hline $60 \mathrm{~min}$ & $66,33 \pm$ & 2,52 & $6,00 \pm$ & 1,00 & $21,00 \pm$ & 3,61 & $6,67 \pm$ & 0,58 & $0,00 \pm$ & 0,00 \\
\hline $360 \mathrm{~min}$ & $66,00 \pm$ & 2,11 & $5,33 \pm$ & 2,89 & $22,67 \pm$ & 8,02 & $5,67 \pm$ & 3,67 & $0,33 \pm$ & 0,11 \\
\hline \multicolumn{11}{|l|}{$100 \mathrm{mg} / \mathrm{kg}$} \\
\hline $5 \mathrm{~min}$ & $64,67 \pm$ & 3,51 & $6,00 \pm$ & 1,53 & $22,33 \pm$ & 2,08 & $6,67 \pm$ & $1,00^{\mathbf{A B}}$ & $0,33 \pm$ & 0,11 \\
\hline $30 \mathrm{~min}$ & $67,00 \pm$ & 1,73 & $5,33 \pm$ & 0,58 & $23,00 \pm$ & 2,65 & $4,67 \pm$ & 1,15 & $0,00 \pm$ & 0,00 \\
\hline $60 \mathrm{~min}$ & $65,00 \pm$ & 6,24 & $6,00 \pm$ & 1,00 & $23,67 \pm$ & 6,66 & $5,33 \pm$ & 0,58 & $0,00 \pm$ & 0,00 \\
\hline $360 \mathrm{~min}$ & $64,67 \pm$ & 7,02 & $5,67 \pm$ & 1,53 & $24,00 \pm$ & 9,17 & $5,67 \pm$ & 1,53 & $0,00 \pm$ & 0,00 \\
\hline \multicolumn{11}{|l|}{$200 \mathrm{mg} / \mathrm{kg}$} \\
\hline $5 \mathrm{~min}$ & $64,50 \pm$ & 2,12 & $5,50 \pm$ & 0,71 & $24,00 \pm$ & 2,83 & $6,00 \pm$ & $0,00^{\mathbf{A B}}$ & $0,00 \pm$ & 0,00 \\
\hline $30 \mathrm{~min}$ & $61,33 \pm$ & 2,31 & $6,33 \pm$ & 1,53 & $28,00 \pm$ & 2,00 & $4,33 \pm$ & 0,58 & $0,00 \pm$ & 0,00 \\
\hline $60 \mathrm{~min}$ & $66,33 \pm$ & 2,08 & $4,33 \pm$ & 0,58 & $23,00 \pm$ & 1,00 & $6,33 \pm$ & 1,53 & $0,00 \pm$ & 0,00 \\
\hline $360 \min$ & $66,33 \pm$ & 2,08 & $6,00 \pm$ & 2,00 & $21,00 \pm$ & 3,61 & $6,37 \pm$ & 2,00 & $0,33 \pm$ & 0,11 \\
\hline \multicolumn{11}{|l|}{$400 \mathrm{mg} / \mathrm{kg}$} \\
\hline $5 \mathrm{~min}$ & $69,67 \pm$ & 3,21 & $5,33 \pm$ & 1,15 & $19,67 \pm$ & 2,52 & $5,33 \pm$ & $1,15^{\mathbf{B}}$ & $0,00 \pm$ & 0,00 \\
\hline $30 \mathrm{~min}$ & $67,00 \pm$ & 1,00 & $5,33 \pm$ & 1,53 & $22,67 \pm$ & 3,06 & $5,00 \pm$ & 1,00 & $0,00 \pm$ & 0,00 \\
\hline $60 \mathrm{~min}$ & $62,67 \pm$ & 1,53 & $6,00 \pm$ & 2,00 & $26,00 \pm$ & 4,36 & $5,33 \pm$ & 1,53 & $0,00 \pm$ & 0,00 \\
\hline $360 \min$ & $58,33 \pm$ & 0,58 & $5,00 \pm$ & 1,00 & $31,33 \pm$ & 1,53 & $5,33 \pm$ & 1,15 & $0,00 \pm$ & 0,00 \\
\hline \multicolumn{11}{|l|}{$800 \mathrm{mg} / \mathrm{kg}$} \\
\hline $5 \min$ & $70,00 \pm$ & 3,61 & $4,33 \pm$ & 0,58 & $21,33 \pm$ & 3,51 & $4,33 \pm$ & $0,58^{\mathbf{B}}$ & $0,00 \pm$ & 0,00 \\
\hline $30 \mathrm{~min}$ & $65,67 \pm$ & 3,51 & $5,67 \pm$ & 1,53 & $23,00 \pm$ & 4,58 & $5,67 \pm$ & 2,08 & $0,00 \pm$ & 0,00 \\
\hline $60 \mathrm{~min}$ & $66,00 \pm$ & 2,65 & $5,67 \pm$ & 2,08 & $22,67 \pm$ & 1,53 & $5,67 \pm$ & 1,53 & $0,00 \pm$ & 0,00 \\
\hline $360 \min$ & $61,33 \pm$ & 2,52 & $4,33 \pm$ & 0,58 & $30,00 \pm$ & 2,65 & $4,33 \pm$ & 0,58 & $0,00 \pm$ & 0,00 \\
\hline \multicolumn{11}{|l|}{$1600 \mathrm{mg} / \mathrm{kg}$} \\
\hline $5 \mathrm{~min}$ & $67,33 \pm$ & 5,69 & $5,33 \pm$ & 1,53 & $23,33 \pm$ & 5,77 & $4,00 \pm$ & $0,00^{\mathbf{B}}$ & $0,00 \pm$ & 0,00 \\
\hline $30 \mathrm{~min}$ & $65,33 \pm$ & 1,53 & $4,67 \pm$ & 1,15 & $25,33 \pm$ & 1,15 & $4,67 \pm$ & 0,58 & $0,00 \pm$ & 0,00 \\
\hline $60 \mathrm{~min}$ & $62,50 \pm$ & 2,12 & $7,00 \pm$ & 1,41 & $25,50 \pm$ & 4,95 & $5,00 \pm$ & 1,41 & $0,00 \pm$ & 0,00 \\
\hline $360 \mathrm{~min}$ & $63,00 \pm$ & 3,46 & $4,67 \pm$ & 1,15 & $27,67 \pm$ & 1,15 & $4,67 \pm$ & 1,15 & $0,00 \pm$ & 0,00 \\
\hline
\end{tabular}

Medias seguidas de letras distintas para el tiempo $5 \mathrm{~min}$, entre tratamientos, presentaron diferencias significativas $(\mathrm{p}<0,05)$

plementación no debe ser excesiva y que niveles de 50 y 100 mg/kg de ácido ascórbico en la dieta son suficientes para garantizar óptimo crecimiento de juveniles de Cyprinus carpio.

Respecto a los parámetros hematológicos como probables indicadores de estrés, se debe anotar que algunos autores reportan un aumento en los valores de hemoglobina y hematocrito como consecuencia de exposición a estrés de varias especies de peces (Chagas y Val, 2003; Zhou et al., 2012). En Colossoma macropomum, Chagas y Val (2003) afirman que la suplementación con ácido ascórbico en la dieta tiene efecto importante sobre algunos de los parámetros hematológicos de la especie, como el hematocrito, el cual es directamente proporcional a los niveles de ácido ascórbico contenido en la dieta, observándose valores reducidos en los ejemplares alimentados con dietas libres de ácido ascórbico. Resultados similares fueron obtenidos en otras especies (Fracalossi et al., 1998; Li y Robinson, 1999; Adham et al., 2000) que verificaron disminución del hematocrito en los ejemplares que no recibieron suplementación con vitamina C. Los resultados de este trabajo difieren de lo descri- 
to anteriormente, ya que no se observaron diferencias significativas entre los tratamientos, ni en los tiempos de hipoxia. En cuanto a la hemoglobina los resultados obtenidos por Adham et al., (2000), difieren con los encontrados por Soliman et al., (1994) y Chagas y Val (2003), quienes reportaron reducción significativa de los niveles de hemoglobina en ejemplares alimentados sin vitamina C. Dicha reducción probablemente se deba a la disminución en la capacidad de absorber y redistribuir el hierro, con la consecuente baja en la capacidad de sintetizar hemoglobina. La disminución de ambos parámetros, podrían indicar estados de anemia de los animales (Adham et al., 2000), los cuales no fueron observados en el presente estudio.

En cuanto al conteo de células sanguíneas Zhou et al., (2012) observaron que el contaje de celulas rojas fue mayor en juveniles de Rachycentron canadum suplementados con diferentes niveles de vitamina C cuando se comparó con el conteo obtenido en ejemplares alimentados con dieta libre de la vitamina. Asimismo, Gusmão et al., (2007) encontraron que en juveniles de Brycon amazonicus, el número de leucocitos fue significativamente mayor en ejemplares suplementados con vitamina $\mathrm{C}$ con respecto a los no suplementados, presentando los mayores valores en los tratamientos con 600 y 800 mg/kg. Similares resultados se obtuvieron en el presente estudio, en el cual los ejemplares suplementados con $800 \mathrm{mg} / \mathrm{kg}$ de ácido ascórbico presentaron mayor número de leucocitos debido probablemente a la acción inmunoestimulante que se le ha atribuido a la vitamina C (Narra et al., 2015), según la cual podría mejorar la producción de células blancas en la sangre del organismo haciéndolo más resistente a situaciones adversas. Barros et al., (2014) afirman que en condiciones de estrés, se presenta linfopenia en Tilapia nilotica y que tal situación podría mejorarse al suplementar la dieta con ácido ascórbico (600mg/ kg), lo cual corrobororía tal hallazgo.

Por otro lado, en el presente estudio no se observó relación entre el número de linfocitos circulantes y los niveles de ácido ascórbico suplementado, así como tampoco sobre la producción de neutrófilos. En cuanto a los monocitos, principales células de la respuesta inmune de los peces, se esperaría un aumento ante condiciones de estrés. Sin embargo, en el presente estudio todos los grupos tratados mostraron un evidente un descenso en el primer tiempo de colecta (5 minutos) post estrés con diferencias significativas $(p<0,05)$, al ser comparados con el grupo de no suplementados. Probablemente, tal descenso se deba a una respuesta de atenuación del estrés, la cual por supuesto debe ser verificada con otros parámetros no evaluados en el presente trabajo.
En conclusión, se puede afirmar que la suplementación con niveles de $100 \mathrm{mg} / \mathrm{kg}$ de ácido ascórbico en la dieta de juveniles de Piaractus brachypomus contribuye a mejorar los parámetros productivos (GP, CR y TCE), pero que la utilización de niveles crecientes de vitamina $C$ no afectan la mayoría de parámetros hematológicos de la especie, con excepción del número total de leucocitos, el cual aumenta y el porcentaje de monocitos, cuyo aumento se ve atenuado por el uso de la vitamina.

\section{Referencias}

Abreu JS, Urbinati EC. Physiological responses of matrinxã (Brycon amazonicus) fed different levels of vitamin $\mathrm{C}$ and submitted to air exposure. Acta Amazon. 2006;36:519-524.

Adham GK, Hashem HO, Abu-Shabana MB, Kamel AH. Vitamin $C$ deficiency in the catfish Clarias gariepinus. Aquacult Nutr. 2000;6:129-139.

Ai Q, Mai K, Zhang C, Xu W, Duan Q, Tan B, Liufu Z. Effects of dietary vitamin $C$ on growth and immune response of Japanese seabass, Lateolabrax japonicus. Aquacult. 2004;242:489-500.

AOAC. 2012. Official Method of Analysis of the Association of Analytical Chemists 19 Edition, Association of Official Analytical Chemists. Washington, DC, USA, p. 1018.

Arab N, Islami HR. Effects of Dietary Ascorbic Acid on Growth Performance, Body Composition, and Some Immunological Parameters of Caspian Brown Trout, Salmo trutta caspius. J World Aquacult Soc. 2015;46(5):505-518.

Barnett CW, Pankhurst NW. The effects of common laboratory and husbandry practices on the stress response of greenback flounder Rhombosolea tapirina (Günther, 1862). Aquacult. 1998;162:313-329.

Barros MM, Falcon DR, Orsi RO, Pezzato LE, Fernandes AC Jr., Guimarães IG, Fernandes A Jr., Padovani CR, Sartori MMP. Non-specific immune parameters and physiological response of Nile tilapia fed b-glucan and vitamin C for different periods and submitted to stress and bacterial challenge. Fish Shellfish Immun. 2014;39:188-195.

Barton BA, Iwama GK. Physiological changes in fish from stress en: aquaculture with emphasis on the response and effects of corticosteroids. Annu Rev Fish Dis. 1991;1:3-26.

Chagas EC, Val AL. Effect of vitamin C on weight and hematology of tambaqui. Pesqui Agropecu Bras. 2003;38(3):397-402.

Chen YJ, Yuan RM, Liu YJ, Yang HJ, Liang GY, Tian LX. Dietary vita$\min C$ requirement and its effects on tissue antioxidant capacity of juvenile largemouth bass, Micropterus salmoides. Aquacult. 2015;435:431-436.

Conroy D. 1998. Manual de métodos y técnicas de laboratorio de uso común en la hematología pisciaria. Documento Técnico 1, Pp. 25.

Darias MJ, Mazurais D, Koumoundouros G, Cahu CL, Zambonino JL. Overview of vitamin $\mathrm{D}$ and $\mathrm{C}$ requirements in fish and their influence on the skeletal system. Aquacult. 2011;315:49-60.

Drouin G, Godin JR, Pagé B. The Genetics of Vitamin C Loss in Vertebrates. Curr Genomics. 2011;12:371-378. 
Falcon DR, Barros M, Pezzato LE, Sampaio FG, Hisano H. Physiological Responses of Nile Tilapia, Oreochromis niloticus, Fed vitamin C- and lipid-supplemented diets and dubmitted to lowtmperature stress. J World Aquacult Soc. 2007;38(2):287-295.

FAO. 2018. El estado mundial de la pesca y la acuicultura 2018. Cumplir los objetivos de desarrollo sostenible. Roma. Licencia: CC BY-NC-SA 3.0 IGO. http://www.fao.org/3/I9540ES/ i9540es.pdf pág 128

FAO. 2016. El estado mundial de la pesca y la acuicultura. Contribución a la seguridad alimentaria y la nutrición para todos. Roma, Italia, Pp.224.

Fracalossi D, Allen M, Nichols D, Oftedal O. Oscars, Astronotus ocellatus, have a dietary requirement for vitamin C. J Nutr. 1998;128:1745-1751.

Gao J, Koshio S, Ishikawa M, Yokoyama S, Mamauag RE. Interactive effects of vitamin $C$ and $E$ supplementation on growth performance, fatty acid composition and reduction of oxidative stress in juvenile Japanese flounder Paralichthys olivaceus fed dietary oxidized fish oil. Aquaculture, 2014;422(423) 84-90.

Gusmão E, Costa E, Tavares M, Cruz G, Melo C, Da Silva E. Effect of high levels of dietary vitamin $\mathrm{C}$ on the blood responses of matrinxã (Brycon amazonicus). Comp Biochem Physiol. 2007;147(A):383-388.

Ibrahem MD, Fathi M, Mesalhy S, Abd El-Aty AM. Effect of dietary supplementation of inulin and vitamin $\mathrm{C}$ on the growth, hematology, innate immunity, and resistance of Nile tilapia (Oreochromis niloticus). Fish Shellfish Immun. 2010;29:241-246.

Li MH, Robinson EH. Dietary ascorbic acid requirement for growth and health in fish. J Applc Aquacult. 1999;9(2):53-79.

Liang XP, Li Y, Hou YM, Qiu H, Zhou QC. Effect of dietary vitamin C on the growth performance, antioxidant ability and innate immunity of juvenile yellow catfish (Pelteobagrus fulvidraco Richardson). Aquacult Res. 2017;48:149-160.

Martins ML. Evaluation of the addition of ascorbic acid to the ration of cultived Piaractus mesopotamicus (Characidae) on the infrapopulation of Anacanthorus penilabiatus (Monogenea). Braz J Med Biol Res. 1998;31:655-658.

Ming J, Xieb J, Xub P, Geb X, Liuc W, Yea J. Effects of emodin and vitamin $\mathrm{C}$ on growth performance, biochemical parameters and two HSP70s mRNA expression of Wuchang bream (Megalobrama amblycephala Yih) under high temperature stress. Fish Shellfish Immun. 2012;32:651-661.

Miyasaki T, Sato M, Yoshinak R. Efect of vitamin C on lipid and carnitine metabolism in rainbow trout. Fish Sci. 1995;61:501-506.

Narra MR, Rajender K, Reddy RR, Rao JB, Begum G. The role of vitamin $\mathrm{C}$ as antioxidant in protection of biochemical and haematological stress induced by chlorpyrifos in freshwater fish Clarias batrachu. Chemosphere. 2015;132:172-178.
Navarro RD, Ferreira WM, Ribeiro Filho O, Botion LM, Pereira FKS, Silva RF, Maciel TEF. Desempenho de tilápia do Nilo (oreochromis niloticus) suplementada com vitamina c. Arch Zootec. 2010;59(228):589-596.

Notash S. The Effect of Additive Vitamin C on Growth, Feed Conversion Ratio and Survival Rate of Rainbow Trout (Oncorhynchus Mykiss). Aust J Basic Appl Sci. 2012;6(6):86-89.

Ortuño J, Cuesta A, Esteban MA, Meseguer J. Effect to oral administration of high vitamin $C$ and $E$ dosages on the gilthead seabream Sparus aurata innate immune system. Vet Immunol Immunopathol. 2001;79:167-180.

Ramesha TJ, Reddy HR, Naik AT. Effect of Mg-L-ascorbyl-2-phosphate on growth and survival of common carp (Cyprinus carpio). Karnataka J Agric Sci. 2000;13(1):134-137.

Reis ES, Feiden A, Signor A, zaminhan M, Finkier JK, Boscolo WR. Suplementação de vitamina c na dieta para larvas de jundiá Rhamdia voulezi. Ciênc Anim Bras. 2011;12(1):83-89.

Ricker W. 1975. Computation and interpretation of biological statistics of fish populations. Bulletin of the Fisheries Research board of Canada, 191 Pp. 382.

Sloman KA, Taylor AC, Metcalfe NB, Gilmour KM. Stress from air emersion fails to alter chloride cell numbers in the gills of rainbow trout. J Fish Biol. 2001;59:186-190.

Soliman AK, Jauncey K, Roberts RJ. Water-soluble vitamin requirements of tilapia: ascorbic acid (vitamin C) requirement of Nile tilapia, Oreochromis niloticus. Aquacult Fish Manage. 1994;25(3):269-278.

Urbinati EC, Carneiro PCF. 2005. Práticas de manejo e estresse dos peixes em piscicultura. In: Cyrino, JEP, Tópicos especiais en: Cyrino JEP, Urbinati EC, Fracalossi DM, et al. (Editores). Tópicos especiais em piscicultura de água doce tropical intensiva. Sociedade Brasileira de Aqüicultura e Biologia Aquática. Jaboticabal. Brasil. p. 171-193.

Valenzuela A, Alveal K, Tarifeno E. Respuestas hematológicas de truchas (Oncorhynchus mykiss Walbaum 1792) sometidas a estrés hipóxico agudo: serie roja. Gayana. 2002;66(2):255-261.

Verlhac V, Obach A, Gabaudan J, Schüep W, Hole R. Immunomodulation by dietary vitamin $\mathrm{C}$ and glucan in rainbow trout (Oncorhynchus mykiss). Fish Shellfish Immunol. 1998;8:409-424.

Wendelaar-Bonga SE. The stress response in fish. Physiol Rev. 1997;77(3):591-625.

Zaminham M, Luchesi JD, Costa JM, Fries EM, Boscolo WR, Feiden A. Efeito da vitamina $C$ sobre os parâmetros hematológicos de kinguio (Carassius auratus). Rev Bras Cienc Agrar. 2012;7(2):352-357.

Zhou Q, Wang L, Wang H, Xiea F, Wang T. Effect of dietary vitamin $\mathrm{C}$ on the growth performance and innate immunity of juvenile cobia (Rachycentron canadum). Fish Shellfish Immunol. 2012;32:969-975. 